\title{
Vitamine A et vieillissement cérébral
}

Véronique PALLET

Valérie ENDERLIN

\author{
UMR 1286, Nutrition and Integrative \\ Neurobiology, Inra-Université de \\ Bordeaux F-33076 France \\ <veronique.pallet@enscbp.fr>
}

\begin{abstract}
To date, convergent data on the role of retinoic acid in the mature brain have established that this molecule, which acts as a hormone, helps to preserve cerebral plasticity by controlling dendritic spine density as well as hippocampal neurogenesis. Deterioration in cerebral plasticity seems to be at the base of the cognitive decline disease. Furthermore, the transcription of several genes, known as muted, in Alzheimer's patients and whose transcripts are involved in the formation of senile plaques, are controlled by retinoic acid. As seen in other nutrients, aging leads to a lower production of retinoic acid; a phenomenon probably accentuated by the fact that Western populations consume an insufficient amount of vitamin $A$ ( $60 \%$ of the population has a consumption lower than the recommendations). These two phenomena (i.e. level of consumption, the lack of activation of vitamin A) accompanied by important individual differences, would help to explain why some patients have an almost normal aging process, whereas others gradually develop cognitive disorders and then, the disease. A better understanding of the role of a collapse of the retinoid status in the genesis of Alzheimer lesions could, beyond the definition of a preventive nutritional strategy, open therapeutic perspectives, through the use of molecules targeting the nuclear receptors.
\end{abstract}

Key words: aging, brain, memory, Retinoic acid nuclear receptors (RAR, RXR), Alzheimer's disease

pour des fonctions cérébrales optimales, telles que la plasticité synaptique, l'apprentissage et la mémoire. Plus récemment encore, des données provenant de plusieurs études mettent en évidence l'implication de la voie de signalisation des rétinoïdes dans l'étiologie de la maladie d'Alzheimer (MA).

\section{Métabolisme et transport de la vitamine $A$}

Pour les mammifères supérieurs la vitamine A provient exclusivement de l'alimentation : soit sous forme de vitamine préformée (dans sa forme majoritaire il s'agit de rétinol estérifié par des acides gras à longues chaînes comme le palmitate de rétinyle par exemple) dans les produits animaux, ou bien sous forme de caroténoïdes provitaminiques tels que le $\beta$-carotène, $\alpha$-carotène, $\beta$-cryptoxanthine, présents dans les aliments d'origine végétale (figure 1). On recom- mande actuellement que $60 \%$ de l'apport en vitamine A soit sous la forme de caroténoïdes (sources végétales) et $40 \%$ sous forme de rétinol (sources animales) (Cordain et al., 2005). L'ester de rétinol (RE) doit être hydrolysé avant d'être absorbé au niveau intestinal. L'efficacité d'absorption est meilleure pour la vitamine $\mathrm{A}$ préformée (80 à $90 \%)$ que pour les caroténoïdes (50$60 \%$ ). Le carotène est converti en rétinol au niveau de la muqueuse intestinale. Le rétinol est ensuite estérifié dans les cellules de la muqueuse par la LRAT (Lecithin : retinol acyltransférase), le RE résultant de cette catalyse est incorporé dans les chylomicrons et absorbé via le système lymphatique (Harrison, 2005). Dans des conditions nutritionnelles normales, la plupart de la vitamine A de l'organisme est stockée dans le foie (essentiellement sous forme de rétinyl ester: RE) pour une part dans les hépatocytes et pour la majorité sous forme de gouttelettes lipidiques dans les cellules étoilées du foie (encore appelées

Pour citer cet article : Pallet V, Enderlin V. Vitamine A et vieillissement cérébral. OCL $2011 ; 18(2)$ : 68-75. doi : 10.1684/ocl.2011.0375 


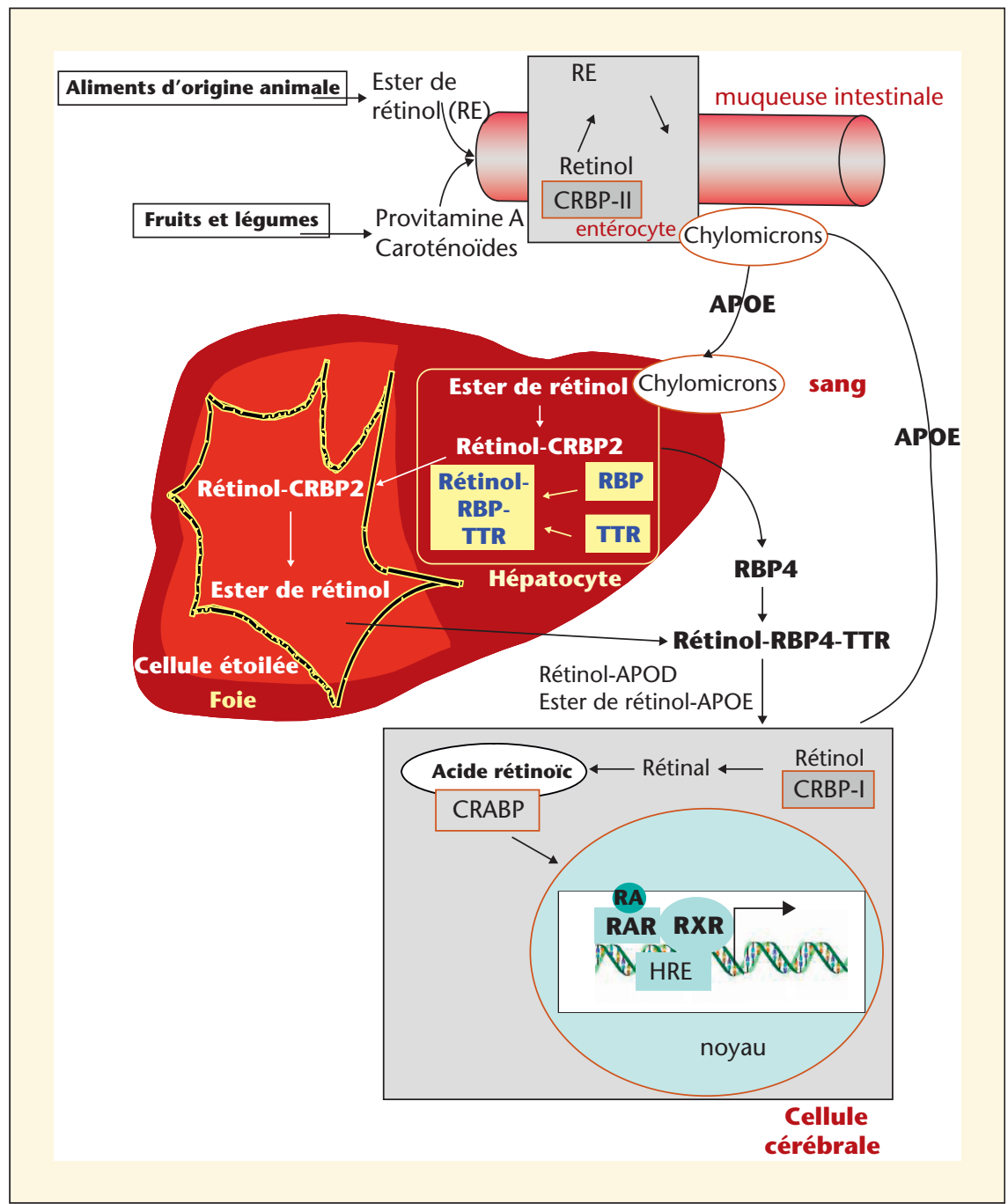

Figure 1. Métabolisme et action cellulaire de la vitamine A.

cellules de Ito). De là, en fonction des besoins de l'organisme, elle sera mobilisée vers les tissus cibles (Bellovino et al., 2003). Pour ce faire, le $\mathrm{RE}$ est hydrolysé et le rétinol libre est ensuite complexé à la retinol binding protein (RBP4). Approximativement $95 \%$ de la RBP circule dans le plasma sous forme d'un complexe avec la protéine de transport de la thyroxine : la transthyretin ou préalbumine (TTR). Le rétinol constitue le rétinoïde le plus abondant dans le sang (95\% du rétinol sont liés aux protéines vectrices permettant leur transport). Le flux de rétinol libéré par le foie est très finement régulé de manière à maintenir une concentration constante de rétinol dans le plasma $(2 \mu \mathrm{mol} / \mathrm{L})$. Au-delà des besoins immédiats, la vitamine A alimentaire sert mière oxydation est réversible, tandis que la deuxième est irréversible. Notons que des voies cytosoliques et microsomales dans les processus d'oxydation du rétinol en $A R$ ont été décrites. La formation du composé intermédiaire, le rétinal, peut être catalysée par des enzymes cytosoliques ou microsomales appelées rétinol déshydrogénases (RDH) (Gottesman et al., 2001 ; Pares et al., 2008). Ces RDH peuvent ainsi appartenir à la famille des alcool déshydrogénases $(A D H)$, enzymes cytosoliques, ou à la famille des déshydrogénases/réductases à chaînes courtes (SDR), enzymes microsomales (Pares et al., 2008). Le rétinal peut ensuite être converti de manière irréversible en AR par des aldéhydes déshydrogénases (ALDH) cytosoliques (Chen et al., 1994). Par ailleurs, il a également été montré, in vitro, qu'une variété de cytochromes P450 (CYP) étaient impliqués dans l'oxydation microsomale du rétinal en AR (Zhang et al., 2000). Enfin, signalons que la CYP26 permet la dégradation et I'élimination de I'AR tout-trans spécifiquement, et contribue ainsi au contrôle du signal rétinoïde (Petkovich, 2001).

\section{Les récepteurs nucléaires de I'acide rétinoïque}

Le fait que I'AR active des facteurs de transcription nucléaire a été découvert en 1987. II existe deux types de récepteurs de I'AR (Mangelsdorf, 1994). Le premier type comprend les récepteurs RAR (retinoic acid receptor) qui peuvent lier I'AR tout-trans et I'AR 9-cis. Le deuxième type comprend les RXR (retinoid $X$ receptor) découverts par Mangelsdorf et al. (1990), dont l'affinité est élevée pour l'AR 9-cis, mais plus faible pour l'AR tout-trans (Levin et al., 1992) (Chambon, 1996). Plus récemment, il a été démontré que certains acides gras, dont le DHA (acide docosahexaénoïque $\mathrm{C} 22: 6 \mathrm{n}-3)$ sont également des ligands du RXR, qu'ils lient avec une affinité comparable à l'acide rétinoïque luimême (de Urquiza et al., 2000). Pour chacun de ces deux récepteurs (RAR et RXR), trois types de protéines, codées par trois gènes différents, ont été isolées : $\operatorname{RAR} \alpha, \beta, \gamma$ et $\operatorname{RXR} \alpha, \beta, \gamma$ (Chambon, 1996).

Ces récepteurs appartiennent à une superfamille de récepteurs nucléaires qui sont des protéines transrégulatrices capables de se fixer principalement sous 
forme d'homodimères de RXR, ou $\mathrm{d}$ 'hétérodimères RAR-RXR au niveau de site spécifiques de I'ADN, appelés éléments de réponse (RXRE et RARE) et situés en amont du gène cible (occasionnellement dans les introns) (Mangelsdorf et Evans, 1995). Il existe une forte homologie de structure entre les membres de la famille des récepteurs nucléaires, qui comprend les récepteurs de différents ligands hydrophobes tels que les hormones stéroïdes, les hormones thyroïdiennes, les dérivés hydroxylés de la vitamine $D$, I'acide rétinoïque ou encore les acides gras et eicosanoïdes (Huang et al., 2010). Cependant, certains récepteurs $n^{\prime}$ ont pas encore à ce jour de ligands connus, ce sont les récepteurs dits orphelins. En présence de leur ligand et en association avec des co-activateurs ou co-represseurs, ces récepteurs régulent positivement ou négativement l'expression de leurs gènes cibles. RAR se lie principalement au RARE sous forme de dimère avec le RXR qui lui agit indépendamment de son ligand. II semble que le dimère RAR/ $R X R$ régule environ 500 gènes (Blomhoff et Blomhoff, 2006).

Notons que RXR joue un rôle central dans la régulation génique puisqu'il est considéré comme le partenaire commun de dimérisation d'autres membres de la superfamille, dont les PPAR (récepteurs des acides gras) (Germain et al., 2006). Ainsi, I'hétérodimérisation du RXR avec les différents membres de la superfamille implique une interaction, voire une compétition, entre les différentes voies de signalisation nucléaires.

Certains auteurs ont montré que I'AR peut également agir selon une voie non génomique. Cette action ultra-rapide participerait à la modulation de la formation des spinules dans la rétine, à la régulation des GAP jonctions et aurait des effets sur les épines dendritiques dans I'hippocampe.

\section{La vitamine $A$ et le cerveau adulte}

\section{La voie de signalisation de I'acide rétinoïque dans le cerveau}

Au cours du développement, la vitamine A et plus précisément l'acide rétinoïque joue un rôle clé dans la structuration cérébrale, la neurogenèse, l'adressage neuronal, la poussée des neurites. Des études récentes rapportent que les rétinoïdes jouent également un rôle important dans le système nerveux central adulte (Lane et Bailey, 2005 ; Bremner et McCaffery, 2007 ; Tafti et Ghyselinck, 2007) en particulier dans des régions où la plasticité neuronale est très importante, i.e. I'hippocampe, le cortex préfrontal médian ainsi que les régions rétrospléniales. Un ensemble de données montre également l'importance de l'acide rétinoïque dans le fonctionnement du striatum et du noyau accubems. D'autres travaux ont montré que le striatum synthétise l'acide rétinoïque et contient toute la machinerie moléculaire associée à son métabolisme et à son activité.

\section{Transport vers le cerveau}

À ce jour, quelques données sont disponibles sur le mode de passage du rétinol à travers la barrière hématoencéphalique (BHE). Yamagata et al. (1993) ont étudié le transport jusqu'au cerveau d'AR injecté dans la cavité péritonéale. $\mathrm{D}^{\prime}$ autres résultats, obtenus dans des conditions d'apports suffisants en vitamine A chez le rat, ont montré que $90 \%$ de l'AR total du cerveau n'est pas synthétisé localement mais provient du pool circulant dans le plasma (Kurlandsky et al., 1995). Par la suite, il a été montré que l'isomère tout-trans de I'AR est la forme la plus largement transportée du sang au cerveau par rapport aux deux autres isoformes, I'AR 13-cis et I'AR 9-cis (Le Doze et al., 2000). Par ailleurs, la présence d'AR a été mise en évidence au niveau cérébral, chez des rats carencés en vitamine $A$ traités avec de I'AR, I'hippocampe et le cortex contenant les proportions les plus importantes (Werner et Deluca, 2002). L'origine de l'AR dans le cerveau semble donc largement exogène dans des situations d'apports suffisants en vitamine A.

\section{Métabolisme cérébral}

Bien que l'apport en AR au niveau cérébral soit majoritairement d'origine exogène, le cerveau adulte possède toute la machinerie nécessaire à la synthèse de I'AR, à son transport et à son action nucléaire (Lane et Bailey, 2005).

L'identification de protéines de liaison des rétinoïdes et des enzymes impliquées dans la biosynthèse de I'AR dans le cerveau adulte plaide en faveur d'un métabolisme cérébral du rétinol. En effet, la présence des protéines de transport CRBP (cellular retinol binding protein) et CRABP (cellular retinoic acid binding protein) (Zetterstrom et al., 1994) (Zetterstrom et al., 1999), des enzymes de conversion ADH1, ADH4 (Martinez et al., 2001) et RALDH (McCaffery et Drager, 1994) a été révélée dans certaines structures du cerveau adulte telles que I'hippocampe et le striatum, structures particulièrement impliquées dans les processus mnésiques.

Outre la mise en évidence des enzymes intervenant dans le métabolisme de la vitamine A, le cerveau adulte est capable de synthétiser l'AR de novo et ce de façon efficace (Dev et al., 1993). La synthèse $d^{\prime} A R$ a en effet été mise en évidence dans le cerveau de souris adulte et plus particulièrement dans le striatum, où cette synthèse serait plus importante que dans I'hippocampe (McCaffery and Drager, 1994). De plus, l'équipe de Sakai et al. (2004) a montré que l'AR est synthétisé par la RALDH2 au niveau des méninges adjacentes à l'hippocampe. Enfin, la présence de rétinol et surtout des rétinyl esters a pu être mise en évidence dans I'hippocampe de cerveau humain mature (Connor et Sidell, 1997).

\section{Modulation de la plasticité cérébrale}

Parmi les nombreux gènes dont l'expression est régulée par l'AR dans le cerveau adulte, il ya ceux codant pour ses propres récepteurs, et ceux codant pour des protéines spécifiques des neurones impliquées dans beaucoup de fonctions du cerveau mature. À titre d'exemples on peut citer : la synaptophysine, le NGF, les récepteurs au $\mathrm{N}$-methyl-D-aspartate (NMDA), le récepteur 2 à la dopamine, la choline acetyltransferase, la neurogranie ou encore la neuromoduline. Enfin, l'acide rétinoïque et ses récepteurs régulent aussi un certain nombre de gènes codant pour des protéines impliquées dans les processus neurodégénératifs telles que I'APP (amyloid protein precursor) ou en encore la protéine tau.

Il est actuellement admis que I'AR joue un rôle dominant dans la préservation des fonctions cérébrales. Ainsi, l'étude des effets du statut en vitamine $A$, ou en acide rétinoïque, dans le cerveau adulte est de la plus grande importance, et en particulier au cours du vieillissement. En effet, des données récentes ont montré que des modifications du statut en rétinoïdes induisent la mise en place d'altérations dans l'expression des protéines neuro- 
nales cibles et en conséquence, affectent le maintien des processus physiologiques dans le cerveau adulte (Malik et al., 2000). Ainsi, des altérations de la plasticité cérébrale et des déficits de mémoire ont été décrits chez l'animal carencé en vitamine $A$. II a aussi été démontré que les souris Knockout pour les récepteurs RAR $\beta$ et $R X R \beta-R X R \gamma$ présentaient une altération de la LTP (potentialisation à long terme, une forme de plasticité synaptique) ainsi que des déficits substantiels des performances mnésiques, mis en évidence dans un test de mémoire spatiale dépendante de I'hippocampe (Chiang et al., 1998; Mingaud et al., 2008). La mutation du RAR $\beta$ avec, soit celle du RXR $\beta$, soit celle du $R X R \gamma$, entraîne des déficits de locomotion caractéristiques $d^{\prime}$ une fonction anormale du striatum et probablement liés à une diminution de l'expression des récepteurs à la dopamine dans les neurones striataux (Krezel et al., 1998 ; Alfos et al., 2001).

L'acide rétinoïque régule aussi l'expression de gènes codant pour des protéines impliquées dans les processus de neurogenèse, telles que les neurotrophines NGF et BDNF, et leurs récepteurs respectifs TrkA et TrkB (Scheibe et Wagner, 1992). Récemment, quelques études se sont intéressées aux effets d'une hypoactivité de la voie des rétinoïdes sur les processus de neurogenèse chez l'adulte, en utilisant la carence nutritionnelle en vitamine A comme modèle d'étude. Ainsi, une augmentation de la prolifération et une diminution de la différenciation ont été observées dans le bulbe olfactif des animaux déficients (AssonBatres et al., 2003). Une diminution de la survie et de la différenciation neuronale a été mise en évidence dans l'hippocampe, d'animaux carencés en vitamine $A$. Cependant, un régime enrichi en vitamine $A$ ne permettait pas de rétablir le niveau de neurogenèse chez les souris carencées (Jacobs et al., 2006). Enfin, des travaux récents ont mis en évidence que la carence en vitamine $A$ induit une altération de la neurogenèse hippocampique (diminution de la prolifération cellulaire, survie cellulaire et différenciation neuronale) parallèlement à une diminution de TrkA. Ces effets étaient réversés par I'administration d'AR touttrans (Bonnet et al., 2008).

\section{Modulation des capacités mnésiques}

La carence vitaminique $A$, induisant un hypoactivité de la voie des rétinoïdes, entraîne chez la souris adulte des déficits cognitifs révélés dans un test de mémoire relationnelle (Etchamendy et al., 2003). De plus, I'administration d'AR à des rats carencés en vitamine A permet de corriger les déficits de mémoire spatiale de référence mesurés dans le labyrinthe aquatique de Morris (Bonnet et al., 2008). D'autres études confortent la relation entre le niveau d'activité de la voie de signalisation des rétinoïdes et les processus cognitifs. Pour exemple, il a été démontré qu'une carence vitaminique $A$ induisait des déficits d'apprentissage et de mémoire spatiale dans un test de labyrinthe radial, et qu'une supplémentation en vitamine A permettait de corriger les déficits observés (Cocco et al., 2002). En revanche, d'autres travaux mettant en œuvre l'isomère 13-cis de I'AR, molécule utilisée sous la dénomination Accutane dans le traitement de l'acnée, ont montré des effets néfastes de cette molécule dans un test de mémoire hippocampo-dépendante chez la souris jeune (Crandall et al., 2004). Ce résultat est à ce jour controversé par l'étude de Ferguson et Berry (2007) qui démontre, au contraire, que le traitement par la forme 13-cis de l'AR est sans effet sur l'apprentissage et la mémoire spatiale chez le rat.

L'ensemble de ces données montre que les situations nutritionnelles ou physiologiques conduisant à un déficit d'activité de la voie de signalisation des rétinoïdes, et en particulier à des modifications de l'expression des récepteurs nucléaires ou de leurs gènes cibles dans le cerveau, conduisent à de considérables dommages neurobiologiques et à des altérations des performances mnésiques.

\section{Vitamine $A$ et vieillissement cérébral}

Une forte perturbation du métabolisme de la vitamine A apparaît au cours du vieillissement. Elle peut conduire à des concentrations élevées de cette vitamine dans le foie (van der Loo et al., 2004) alors que dans le même temps la capacité de l'organisme à mobiliser les réserves hépatiques de rétinol et à les utiliser efficacement semble fortement affectée chez I'homme âgé (Azais-Braesco et al., 1995). Il en résulte une diminution de la biodisponibilité cellulaire en acide rétinoïque qui se traduit chez l'animal âgé, dans plusieurs tissus cibles (foie, cerveau) par une baisse d'activité de la voie de signalisation des rétinoïdes
(Enderlin et al., 1997; Pallet et al., 1997). Cette baisse d'activité des rétinoïdes, dans les tissus cibles, a également été mise en évidence chez I'homme âgé (Feart et al., 2005).

Il est maintenant admis que la baisse d'activité cellulaire de la vitamine A joue un rôle clé dans l'étiologie de déficits mnésiques spécifiques associés au vieillissement et qu'un traitement par l'acide rétinoïque (AR), est à même de restaurer les capacités mnésiques des animaux âgés. Enfin, plus récemment, la démonstration de l'efficacité d'une supplémentation nutritionnelle en vitamine $\mathrm{A}$ chez les animaux adultes, permettant à la fois le maintien de l'activité de la voie de signalisation et la prévention de l'apparition de troubles mnésiques spécifiques liés au vieillissement, a été faite (Mingaud et al., 2008). Il est donc aujourd'hui bien admis qu'il existe une relation étroite entre le niveau d'activité cérébrale de la voie d'action des rétinoïdes, l'expression de gènes cibles codant pour des protéines neuronales impliquées dans certains processus de plasticité (Husson et al., 2004), et les performances mnésiques au cours du vieillissement (Mingaud et al., 2008).

L'ensemble de ces données suggèrent qu'une régulation précise de l'expression des gènes contrôlés par les rétinoïdes est fondamentalement importante pour le fonctionnement optimal du cerveau et pour le maintien des performances de mémoire.

\section{Vitamine $A$ et maladie d'Alzheimer}

La maladie d'Alzheimer (MA) est la démence la plus répandue chez les sujets âgés. C'est une maladie chronique dégénérative caractérisée par la détérioration progressive des fonctions cognitives incluant la mémoire, le jugement, la prise de décision, le langage, l'orientation, etc. Les symptomes cliniques incluent les altérations de plasticité neuronale (e.g. la perte selective des neurones et des synapses) et la formation de plaques séniles extracellulaires constituées de peptides $\beta$-amyloïdes (A $\beta$ ) ainsi que d'enchevêtrements neurofibrillaires intracellulaires.

Récemment, des données issues de plusieurs études séparées apportent des arguments en faveur d'un rôle de la voie de signalisation de l'acide rétinoïque dans l'étiologie de la maladie 
d'Alzheimer. Tout d'abord, Goodman a démontré les liens génétiques entre cette voie de signalisation et la MA, en mettant en évidence que les loci les plus fréquemment trouvés modifiés chez les sujets atteints de la maladie était systèmatiquement situés sur des clusters très proches de gènes codant pour des protéines ayant un rôle majeur dans le métabolisme et la signalisation des rétinoïdes, à savoir: CYP26, RAR $\alpha$, RXR $\beta \gamma$, RXR $\beta$, CRABP-II et RBP par exemple. CYP26 est un cytochrome P450 impliqué dans le catabolisme de I'AR et participant de ce fait au contrôle du niveau d'AR dans les tissus. Une diminution de la concentration de rétinol sérique $a$, par ailleurs, été révélée chez les patients Alzheimer, ainsi qu'une diminution de l'expression et de l'activité de la retinaldéhyde désydrogénase, enzyme impliquée dans la production de I'AR. Une diminution de la biodisponibilité de I'AR liée à l'âge ou à la dérégulation de gènes codant pour des protéines du métabolisme des rétinoïdes et entraînant des altérations dans l'expression de gènes cibles de ceux-ci, pourrait alors être fortement impliquée dans l'étiologie de la forme tardive (ou sporadique) de la MA (Goodman et Pardee 2003 ; Goodman, 2006).

\section{Les transporteurs de la vitamine A et la MA}

L'apolipoprotéine E (ApoE), apolipoprotéine majeure du liquide cerébrospinal, participerait en complément de RBP au transport du rétinol et des rétinyl esters dans le cerveau. L'allèle $\varepsilon 4$ de son gène a été identifié comme un facteur de risque de la $M A$; il semble favoriser l'agrégation des peptides $A \beta$. En revanche, un effet protecteur de l'allèle $\varepsilon 2$ de I'ApoE, connu comme étant le meilleur transporteur des rétinoïdes, a été trouvé dans plusieurs études. Les niveaux de lipocaline, apolipoprotéine $D$, un autre transporteur du rétinol dans le système nerveux central sont augmentés dans les neurones de patients atteints de la MA. Une régulation positive de son expression par l'AR a été observée in vitro.

\section{Vitamine $A$ et enchevètrements neurofibrillaires :}

Parmi les gènes potentiellement régulés par I'AR, on trouve un gène codant pour la protéine tau encore appelée MAPT pour microtubules-associated-protein tau, et qui est la protéine prépondérante dans la formation des enchevêtrements neurofibrillaires.

\section{Vitamine $A$ et $\beta$-amyloïdes ( $A \beta$ )}

La voie biochimique de synthèse des peptides $A \beta$, peptides constituants de la plaque sénile, est une voie pathologique appelée voie amylö̈dogènique (figure 2). Elle comporte deux séquences de clivages endoprotéolytiques successifs de la

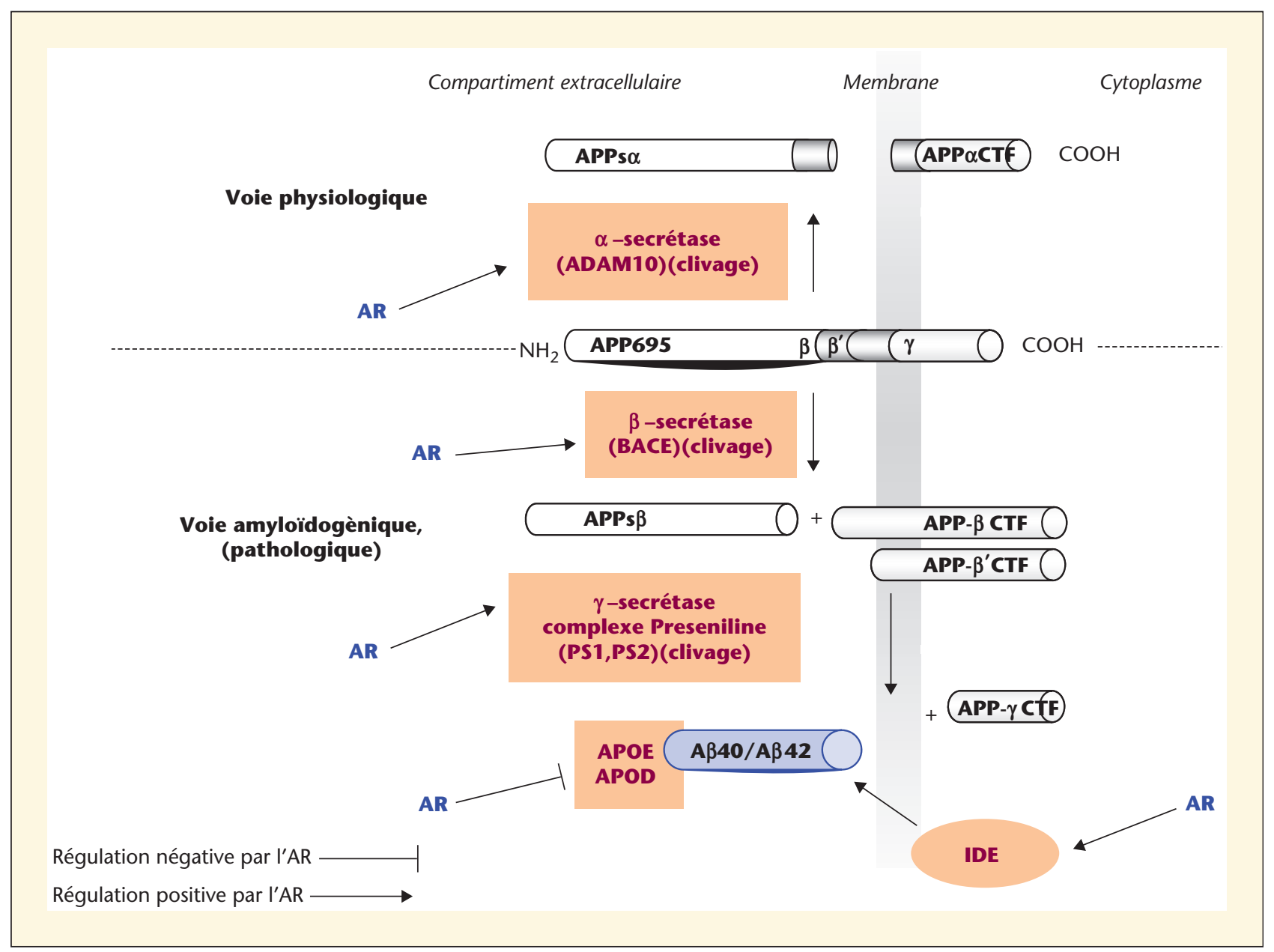

Figure 2. Acide rétinoïque et processus de dégradation de la protéine précurseur du peptide Aß. 
protéine APP (A $\beta$ precursor protein) catalysées par deux protéases distinctes les $\beta$ - et $\gamma$-secrétases. La $\beta$-secrétase ou $\beta$-site cleaving enzyme (BACE) est hautement exprimée dans le cerveau des patients et est localisée sur les sites de production du peptide $A \beta$. Le clivage de I'APP par la $\beta$-secrétase genère des fragments APP $\beta$ dans l'espace extracellulaire. La $\gamma$-secrétase intervient ensuite pour cliver la partie, issue du précédent clivage qui est demeurée dans la membrane. Cette deuxième étape protéolytique produit le petide $A \beta$, le composant central des plaques séniles. En condition physiologique, I'APP peut aussi être protéolysée par une voie non amyloïdogènique. Cette autre voie de dégradation de I'APP comporte une étape protéolytique par une $\alpha$ secrétase, dans la séquence $A \beta$, empêchant ainsi définitivement la production du peptide $A \beta$. Cette activité $\alpha$ secrétase est attribuée aux metalloprotéases ADAM9 et ADAM10.

Il a été montré que l'hypoactivité de la voie de signalisation des rétinoïdes entraîne la formation anormale et le dépôt des petides $A \beta$ (Corcoran et al., 2004). Ceci a en particulier été montré chez des rats carencés en vitamine A. Après consommation pendant 1 an d'une alimentation totalement dépourvue de cette vitamine, les animaux présentaient une hypoactivation de lavoie de signalisation de la vitamine $A$, et avaient développé des dépots $\beta$-amyloïdes dans leur cerveau. Des données supplémentaires ont révélé que la carence en vitamine $A$, génératrice d'une diminution de la biodisponibilité de I'AR, induit une activation de la voie amyloïdogènique dans le cortex des rats, structure connue comme étant la première altérée par la maladie (Husson et al., 2006).

La vitamine A ou ses dérivés, par l'intermédiaire des récepteurs, sont également à même $d^{\prime}$ inhiber ou destabiliser les agrégats $A \beta$ préformés, prévenant ainsi la formation des plaques (Ono et al., 2004) (Sahin et al., 2005). Il y a de nombreuses données biochimiques qui vont dans le sens de l'implication de la voie de signalisation de la vitamine $A$ dans la formation de $A \beta$. En effet, comme on le voit sur la figure 2, les étapes clés du processus de formation des peptides amyloïdes sont sous le contrôle de protéines dont l'expresssion a été montrée in vitro, comme étant régulée par I'AR. Ceci comprend: APP, la $\beta$ secrétase, les presenilines 1 et 2 (PS1 et PS2), deux protéines du complexe $\gamma$ secrétase ainsi que ADAM10. De manière intéressante, une étude in vitro montre qu'un traitement par I'AR augmente l'expression de ADAM10 au niveau protéique, suggérant ainsi que I'AR induit un basculement dans la dégradation de l'APP, en faveur de la voie $\alpha$ secrétase ou voie dites physiologique.

L'insulin dégrading enzyme (IDE), une mettaloprotéase responsable de la dégradation de l'insuline a été montrée comme jouant un rôle capital dans la dégradation du peptide $A \beta$ à la fois in vitro et in vivo. IDE a été mis en évidence dans le liquide cérébrospinal. Son niveau d'activité, la quantité de ses protéine ou ARNm exprimés, sont diminués dans le cerveau des malades et sont associés à une diminution de la quantité de dépôts $A \beta$. Ceci suggère que l'augmentation de I'activité IDE pourrait induire une diminution du risque de développer la MA. Or, le promoteur du gène codant pour IDE présente un élément de réponse aux RAR (RARE), zone préférentielle de fixation des récepteurs de l'AR, et la transcription de IDE est régulée positivement par I'AR.

Enfin, des travaux récents laissent supposer que I'AR pourrait être considéré comme un agent thérapeutique potentiel pour le traitement de la MA. L'administration d'AR à des souris transgèniques développant les lésions de types Alzheimer induit, en effet, une importante diminution des dépots amyloïdes et des enchevêtrements neurofibrillaires (Ding et al., 2008).

\section{Conclusion}

L'ensemble de ces données suggère qu'une régulation très précise de l'expression des gènes médiée par les rétinoïdes est cruciale pour un fonctionnement cérébral optimal, et apporte des arguments en faveur d'un rôle important de la vitamine $A$, via ses récepteurs nucléaires dans les multiples processus impliqués dans la formation des plaques séniles.

Dans une perspective de prévention nutritionnelle de la maladie d'Alzheimer, il sera nécessaire de mieux comprendre l'implication de l'hypoactivité de la voie de signalisation des rétinoïdes se mettant en place naturellement au cours du vieillissement, dans la genèse des lésions pathologiques.

\section{RÉFÉRENCES}

Alfos S, Boucheron C, Pallet V, et al. A retinoic acid receptor antagonist suppresses brain retinoic acid receptor overexpression and reverses a working memory deficit induced by chronic ethanol consumption in mice. Alcohol Clin Exp Res $2001 ; 25$ : 1506-14.

Asson-Batres MA, Zeng MS, Savchenko V, Aderoju A, McKanna J. Vitamin A deficiency leads to increased cell proliferation in olfactory epithelium of mature rats. / Neurobiol $2003 ; 54: 539-54$.

Azais-Braesco V, Moriniere C, Guesne B, et al. Vitamin $A$ status in the institutionalized elderly. Critical analysis of four evaluation criteria: dietary vitamin A intake, serum retinol, relative dose-response test (RDR) and impression cytology with transfer (ICT). Int J Vitam Nutr Res 1995 ; 65 : 151-61.

Bellovino D, Apreda M, Gragnoli S, Massimi M, Gaetani S. Vitamin A transport: in vitro models for the study of RBP secretion. $\mathrm{Mol}$ Aspects Med 2003 ; 24 : 411-20.

Blomhoff R, Blomhoff HK. Overview of retinoid metabolism and function. J Neurobiol 2006 ; $66: 606-30$.

Bonnet E, Touyarot K, Alfos S, Pallet V, Higueret $\mathrm{P}$, Abrous DN. Retinoic acid restores adult hippocampal neurogenesis and reverses spatial memory deficit in vitamin A deprived rats. PLOS ONE $2008 ; 3$ : e3487.

Bremner JD, McCaffery P. The neurobiology of retinoic acid in affective disorders. Prog Neuropsychopharmacol Biol Psychiatry 2007 ; $15: 315-31$.

Chambon P. A decade of molecular biology of retinoic acid receptors. Faseb / $1996 ; 10$ : 940-54.

Chen M, Achkar C, Gudas LJ. Enzymatic conversion of retinaldehyde to retinoic acid by cloned murine cytosolic and mitochondrial aldehyde dehydrogenases. Mol Pharmacol $1994 ; 46: 88-96$.

Chiang MY, Misner D, Kempermann G, et al. An essential role for retinoid receptors RARbeta and RXRgamma in long-term potentiation and depression. Neuron 1998; 21 : 1353-61.

Cocco S, Diaz G, Stancampiano R, et al. Vitamin A deficiency produces spatial learning and memory impairment in rats. Neuroscience 2002 ; 115 : 475-82.

Connor MJ, Sidell N. Retinoic acid synthesis in normal and Alzheimer diseased brain and 
human neural cells. Mol Chem Neuropathol 1997 ; 30 : 239-52.

Corcoran JP, So PL, Maden M. Disruption of the retinoid signalling pathway causes a deposition of amyloid beta in the adult rat brain. Eur J Neurosci 2004 ; 20 : 896902.

Cordain L, Eaton SB, Sebastian A, et al. Origins and evolution of the Western diet : health implications for the 21 st century. Am J Clin Nutr $2005 ; 81$ : 341-54.

Crandall J, Sakai Y, Zhang J, et al. 13-cisretinoic acid suppresses hippocampal cell division and hippocampal-dependent learning in mice. Proc Natl Acad Sci USA 2004 ; $101: 5111-6$

de Urquiza AM, Liu S, Sjoberg $\mathrm{M}$, et al. Docosahexaenoic acid, a ligand for the retinoid $X$ receptor in mouse brain. Science $2000 ; 290: 2140-4$.

Dev S, Adler AJ, Edwards RB. Adult rabbit brain synthesizes retinoic acid. Brain Res 1993 ; 632 : 325-8.

Ding Y, Qiao A, Wang Z, et al. Retinoic acid attenuates beta-amyloid deposition and rescues memory deficits in an Alzheimer's disease transgenic mouse model. / Neurosci $2008 ; 28: 11622-34$.

Enderlin V, Alfos S, Pallet V, et al. Aging decreases the abundance of retinoic acid (RAR) and triiodothyronine (TR) nuclear receptor mRNA in rat brain: effect of the administration of retinoids. FEBS Lett 1997 ; 412 : 629-32.

Etchamendy N, Enderlin V, Marighetto A, Pallet V, Higueret P, Jaffard R. Vitamin A deficiency and relational memory deficit in adult mice: relationships with changes in brain retinoid signalling. Behav Brain Res 2003 ; 145 : 37-49.

Feart C, Mingaud F, Enderlin V, et al. Differential effect of retinoic acid and triiodothyronine on the age-related hypo-expression of neurogranin in rat. Neurobiol Aging $2005 ; 26: 729-38$.

Ferguson SA, Berry KJ. Oral Accutane (13-cisretinoic acid) has no effects on spatial learning and memory in male and female Sprague-Dawley rats. Neurotoxicol Teratol 2007 ; 29 : 219-27.

Germain P, Chambon P, Eichele G, et al. International Union of Pharmacology. LXIII. Retinoid X receptors. Pharmacol Rev 2006 ; $58: 760-72$.

Goodman AB. Retinoid receptors, transporters, and metabolizers as therapeutic targets in late onset Alzheimer disease. I Cell Physiol 2006 ; 209 : 598-603.

Goodman AB, Pardee AB. Evidence for defective retinoid transport and function in late onset Alzheimer's disease. Proc Natl Acad Sci USA 2003 ; 100 : 2901-5.

Gottesman ME, Quadro L, Blaner WS. Studies of vitamin A metabolism in mouse model systems. Bioessays 2001 ; 23 : 409-19.

Harrison EH. Mechanisms of digestion and absorption of dietary vitamin A. Annu Rev Nutr $2005 ; 25: 87-103$.

Huang P, Chandra V, Rastinejad F. Structural overview of the nuclear receptor superfamily: insights into physiology and therapeutics. Annu Rev Physiol 2010 ; 72 : 24772.

Husson M, Enderlin V, Alfos S, Boucheron C, Pallet V, Higueret P. Expression of neurogranin and neuromodulin is affected in the striatum of vitamin A-deprived rats. Mol Brain Res 2004 ; $123:$ 7-17.

Husson M, Enderlin V, Delacourte A, et al. Retinoic acid normalizes nuclear receptor mediated hypo-expression of proteins involved in beta-amyloid deposits in the cerebral cortex of vitamin A deprived rats. Neurobiol Dis 2006 ; $23: 1-10$.

Jacobs S, Lie DC, DeCicco KL, et al. Retinoic acid is required early during adult neurogenesis in the dentate gyrus. Proc Natl Acad SCi USA 2006 ; 103 : 3902-7.

Kawaguchi R, Yu J, Honda J, et al. A membrane receptor for retinol binding protein mediates cellular uptake of vitamin $A$. Science 2007 ; 315 : 820-5.

Krezel W, Ghyselinck N, Samad TA, et al. Impaired locomotion and dopamine signaling in retinoid receptor mutant mice. Science $1998 ; 279: 863-7$.

Kurlandsky SB, Gamble MV, Ramakrishnan R, Blaner WS. Plasma delivery of retinoic acid to tissues in the rat. / Biol Chem $1995 ; 270$ : 17850-7.

Lane MA, Bailey SJ. Role of retinoid signalling in the adult brain. Prog Neurobiol $2005 ; 75$ : 275-93.

Le Doze F, Debruyne D, Albessard F, Barre L, Defer GL. Pharmacokinetics of all-trans retinoic acid, 13-cis retinoic acid, and fenretinide in plasma and brain of Rat. Drug Metab Dispos $2000 ; 28: 205-8$.

Levin AA, Sturzenbecker LJ, Kazmer S, et al. 9 cis retinoic acid stereoisomer binds and activates the nuclear receptor RXR alpha. Nature 1992 ; 355 : 359-61.

Malik MA, Greenwood CE, Blusztajn JK, Berse $B$. Cholinergic differentiation triggered by blocking cell proliferation and treatment with all-trans-retinoic acid. Brain Res $2000 ; 874$ : 178-85.

Mangelsdorf DJ. Vitamin A receptors. Nutr Rev $1994 ; 52$ : S32-44.
Mangelsdorf DJ, Evans RM. The RXR heterodimers and orphan receptors. Cell $1995 ; 83$ : 841-50.

Mangelsdorf DJ, Ong ES, Dyck JA, Evans RM. Nuclear receptor that identifies a novel retinoic acid response pathway. Nature 1990 ; 345 : 224-9.

Martinez SE, Vaglenova J, Sabria J, Martinez MC, Farres J, Pares X. Distribution of alcohol dehydrogenase mRNA in the rat central nervous system. Consequences for brain ethanol and retinoid metabolism. Eur J Biochem 2001 ; 268 : 5045-56.

McCaffery P, Drager UC. High levels of a retinoic acid-generating dehydrogenase in the meso-telencephalic dopamine system. Proc Natl Acad Sci USA 1994 ; 91 : 7772-6.

Mingaud F, Mormede C, Etchamendy N. Retinoid hyposignaling contributes to agingrelated decline in hippocampal function in short-term/working memory organization and long-term declarative memory encoding in mice. J Neurosci 2008 ; 28 : 279-91.

Ono K, Yoshiike Y, Takashima A, Hasegawa K, Naiki H, Yamada M. Vitamin A exhibits potent antiamyloidogenic and fibril-destabilizing effects in vitro. Exp Neurol $2004 ; 189$ : 380-92.

Pallet V, Azais-Braesco V, Enderlin V. Aging decreases retinoic acid and triiodothyronine nuclear expression in rat liver: exogenous retinol and retinoic acid differentially modulate this decreased expression. Mech Ageing Dev 1997 ; 99 : 123-36.

Pares X, Farres J, Kedishvili N, Duester G. Medium- and short-chain dehydrogenase/ reductase gene and protein families: Medium-chain and short-chain dehydrogenases/reductases in retinoid metabolism. Cell Mol Life Sci 2008 ; 65 : 3936-49.

Petkovich PM. Retinoic acid metabolism. J Am Acad Dermatol 2001 ; 45 : S136-42.

Sahin M, Karauzum SB, Perry G, Smith MA, Aliciguzel $Y$. Retinoic acid isomers protect hippocampal neurons from amyloid-beta induced neurodegeneration. Neurotox Res $2005 ; 7: 243-50$.

Sakai Y, Crandall JE, Brodsky J, McCaffery P. 13-cis Retinoic acid (accutane) suppresses hippocampal cell survival in mice. Ann $N$ Y Acad Sci 2004 ; 1021 : 436-40.

Scheibe RJ, Wagner JA. Retinoic acid regulates both expression of the nerve growth factor receptor and sensitivity to nerve growth factor. J Biol Chem 1992 ; 267 : 17611-6.

Tafti M, Ghyselinck NB. Functional implication of the vitamin A signaling pathway in the brain. Arch Neurol 2007; 64 : 1706-11. 
Theodosiou M, Laudet V, Schubert M. From carrot to clinic : an overview of the retinoic acid signaling pathway. Cell Mol Life Sci 2010 ; 67 : 1423-45.

van der Loo B, Labugger R, Aebischer CP, et al. Age-related changes of vitamin A status. J Cardiovasc Pharmacol 2004 ; 43 : 26-30.

Werner EA, Deluca HF. Retinoic acid is detected at relatively high levels in the CNS of adult rats. Am I Physiol Endocrinol Metab 2002 ; 282 : E672-8.
Yamagata T, Momoi T, Kumagai $H$, Nishikawa T, Yanagisawa M, Momoi $M$. Distribution of retinoic acid receptor beta proteins in rat brain: up-regulation by retinoic acid. Biomedical Research 1993 ; $14: 183-90$.

Zetterstrom RH, Simon A, Giacobini MM, Eriksson U, Olson L. Localization of cellular retinoid-binding proteins suggests specific roles for retinoids in the adult central nervous system. Neuroscience $1994 ; 62$ : 899-918.
Zetterstrom RH, Lindqvist E, Mata de Urquiza $\mathrm{A}$, et al. Role of retinoids in the CNS: differential expression of retinoid binding proteins and receptors and evidence for presence of retinoic acid. Eur J Neurosci $1999 ; 11: 407-16$.

Zhang QY, Dunbar D, Kaminsky L. Human cytochrome P-450 metabolism of retinals to retinoic acids. Drug Metab Dispos 2000 ; 28 : 292-7. 